\title{
What Did the Surgical Community Learn from COVID-19 Lockdown?
}

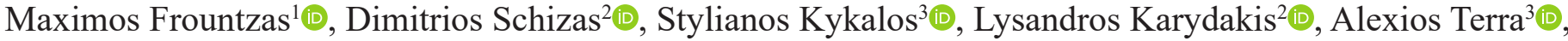 \\ Konstantinos G. Toutouzas ${ }^{1}$
}

\begin{abstract}
${ }^{1}$ First Propaedeutic Department of Surgery, Hippocration General Hospital, National and Kapodistrian University of Athens, School of Medicine, Athens, Greece ${ }^{2}$ First Department of Surgery, Laikon General Hospital, National and Kapodistrian University of Athens, School of Medicine, Athens, Greece ${ }^{3}$ Second Department of Propaedeutic Surgery, Laikon General Hospital, National and Kapodistrian University of Athens, School of Medicine, Athens, Greece
\end{abstract}

To the Editor,

The third outburst of the COVID-19 pandemic seems to be underway. However, restrictive measures against COVID-19 have defined a new landscape in general surgery since its first outburst. COVID-19 reached Greece late during the global pandemic. However, due to concerns that the healthcare system would become overwhelmed, the Greek Government announced a complete lockdown on March 23, 2020. ${ }^{1}$ Also, functional operating rooms were reduced over $50 \%$ due to the transposition of expertized medical staff (anesthesiologists, critical care nurses) to COVID-19 intensive care units (ICUs). The remaining operating rooms were available only for patients with rapidly progressive cancers and emergent operations. ${ }^{2}$ Two COVID-19 referral hospitals were defined, but none of these was accepting surgical cases. Our study aimed to investigate the impact of COVID-19 lockdown on the amount and profile of surgeries during COVID-19 outburst.

Patients that underwent a surgical operation at 3 university surgical departments of tertiary hospitals (not referral for COVID-19) in Greece from January 27, 2020 until June 21, 2020 were included in our study and were divided into 3 groups regarding the period of operation: "pre-lockdown" (January 27, 2020 to March 15, 2020), "lockdown" (March 16, 2020 to May 3, 2020), and "post-lockdown" (May 4, 2020 to June 21, 2020). According to the present study's design, the "pre-lockdown" period has been considered as representative of the condition before the COVID-19 outburst. The study was performed according to the World Medical Association Declaration of Helsinki and ethical approval had been received by the institutional ethics committees. Our study was based on the evaluation of the clinical data of patients that were kept in record after informed consent obtainment, according to the institutions' policy. Statistical analyses were performed using SPSS version 25.0 software for Macintosh (IBM SPSS Corp.;
Armonk, NY, USA). The normality distribution of the numeric variables was tested with the One-sample Kolmogorov Smirnov test. Comparisons of numeric data were conducted with the Kruskal-Wallis test and comparisons of categorical data were performed using the chi-square test. Chi-square test with Bonferroni correction was used to compare emergent surgeries and surgeries for malignancy before and after the lockdown. Statistical significance was considered at a level of $P<.05$.

Our study included 1124 patients, 544 (48\%) male and 580 (52\%) female, with a mean age of $60 \pm 16$ years. The percentage of male patients that underwent surgery were increased during lockdown $(P=0.013)$. Elective operations were significantly decreased during quarantine and after it $(P<.001)$. Surgeries due to malignancy were also reduced considerably during quarantine and after it $(P<.001)$. Also, the majority $(55 \%)$ of surgeries before lockdown were elective for benign diseases, while during the lockdown, the majority (52\%) of surgeries were elective of malignant illnesses $(P<.001)$. The portion of emergent surgeries for malignancy among total operations presented a slight increase during quarantine $(6 \%)$. Moreover, neoadjuvant therapy receivers were increased during and after lockdown (Table $1, P=.016$ ). "Finally, a reduction in the amount of emergent surgeries and surgeries for malignancy was observed before and after the lockdown, but there was no statistically significant difference $(P=.266$ and $P=.139$, respectively)."

During the COVID-19 outburst, access to surgical treatment was reduced for surgical oncologic patients, and alternative therapeutic options, such as endoscopic resections or neoadjuvant therapy, were suggested when possible by oncology associations for the management of gastrointestinal tumors. ${ }^{3}$ Also, fewer emergency surgical admissions, frailer surgical patients, and increased overall 30-day mortality were demonstrated due

Address for Correspondence: Maximos Frountzas, First Propaedeutic Department of Surgery, Hippocration General Hospital, National and Kapodistrian University of Athens, School of Medicine, Athens, Greece

e-mail: froumax@hotmail.com

Received: January 24, 2021 Accepted: April 8, $2021 \cdot$ DOI: 10.5152/balkanmedj.2021.21185

Available at www.balkanmedicaljournal.org

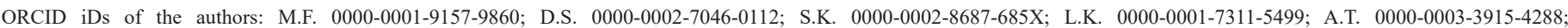
K.G.T. 0000-0002-7830-6945.

Cite this article as:

Frountzas M, Schizas D, Kykalos S, Karydakis L, Terra A, Toutouzas K.G. What did the surgical community learn from COVID-19 lockdown?. [published online ahead of print, 2021 June 16]. Balkan Med J. 2021;38(4):255-256.

Copyright@Author(s)-Available online at http://balkanmedicaljournal.org/ 
TABLE 1. The Effect of COVID-19 Lockdown on Surgical Indications and Patients' Characteristics in Greece

\begin{tabular}{|c|c|c|c|c|}
\hline & Pre-lockdown $(n=546)$ & Lockdown $(n=195)$ & Post-lockdown $(n=383)$ & $P$ \\
\hline Gender & & & & .013 \\
\hline Male & $242(44 \%)$ & $109(56 \%)$ & $193(50 \%)$ & \\
\hline Female & $304(56 \%)$ & $86(44 \%)$ & $190(50 \%)$ & \\
\hline Age (years) & $59 \pm 16(17-94)$ & $62 \pm 17(17-94)$ & $60 \pm 16(15-91)$ & .057 \\
\hline Surgery category & & & & $<.001$ \\
\hline Elective & $461(84 \%)$ & $133(68 \%)$ & $306(80 \%)$ & \\
\hline Emergent & $85(16 \%)$ & $62(32 \%)$ & $77(20 \%)$ & \\
\hline Disease & & & & $<.001$ \\
\hline Benign & $362(66 \%)$ & $81(42 \%)$ & $229(60 \%)$ & \\
\hline Malignant & $184(34 \%)$ & $114(58 \%)$ & $154(40 \%)$ & \\
\hline Indication & & & & $<.001$ \\
\hline Elective benign & $299(55 \%)$ & $31(16 \%)$ & $169(44 \%)$ & \\
\hline Elective malignant & $162(29 \%)$ & $102(52 \%)$ & $136(36 \%)$ & \\
\hline Emergent benign & $63(12 \%)$ & $50(26 \%)$ & $60(16 \%)$ & \\
\hline Emergent malignant & $22(4 \%)$ & $12(6 \%)$ & $18(4 \%)$ & \\
\hline Neoadjuvant therapy & $14 / 162(9 \%)$ & $18 / 102(18 \%)$ & $27 / 136(20 \%)$ & .016 \\
\hline Neoadjuvant interval (days) & $51 \pm 21$ & $48 \pm 17$ & $59 \pm 30$ & .494 \\
\hline
\end{tabular}

*Statistical significance was considered at a level of $P<.05$.

to COVID-19 quarantine, a phenomenon called "lockdown effect."4 A reduction in emergency general surgery admissions was also highlighted in Italy, which was harshly hit by the global coronavirus pandemic. ${ }^{5}$ Also, decreased surgical practices and emergency surgical admissions, with a concomitant increase in morbidity and mortality, were observed in India and South Africa during the first COVID-19 outburst. ${ }^{6,7}$ Greece maintained the fourth-lowest case-fatality ratio worldwide $(5.3 \%)$, which is relatively low compared to other Mediterranean countries. This resulted from timely and strict policy interventions, such as social distancing restrictions and increased ICU beds, that averted 4360 (95\% CI: 3050, 5700) deaths and prevented the healthcare system from becoming overwhelmed. ${ }^{8}$

The lockdown against COVID-19 in Greece seemed to be effective in confining the transmission of the pandemic. Nevertheless, our study demonstrated that COVID-19 quarantine significantly impacted the amount and type of general surgery operations. Therefore, the surgical community should define specific recommendations about the management of benign and malignant surgical conditions and adapt to a new landscape that would last until remission of the pandemic, considering large-scale clinical trials worldwide. ${ }^{9}$ Also, authorities should support health care systems not only in fighting COVID-19 but toward offering high-quality surgical services as well.

Ethics Committee Approval: Ethics committee approval was received for this study from Institutional Review Board of "Hippocration" General Hospital of Athens (Number: 5/55-2021).

Patient Consent for Publication: Informed consent was obtained from the patients.
Author Contributions: Concept - M.F.; Design - D.S.; Supervision - K.T.; Resources A.T.; Materials - S.K.; Data Collection and/or Processing - L.K.; Analysis and/or Interpretation - D.S.; Literature Review - A.T.; Writing - M.F.; Critical Review - K.T.

Conflict of Interest: The authors have no conflicts of interest to declare.

Funding: The authors declared that this study had received no financial support.

\section{REFERENCES}

1. Greek Prime Minster's Office. Prime Minister Kyriakos Mitsotakis' address to the Greek people; 2020. https://primeminister.gr/2020/03/22/23615.

2. Greek Ministry of Health. Surgery program adjustment; 2020. https://www.moh.gov. gr/articles/ministry/grafeio-typoy/press-releases/6838-prosarmogh-programmatos-x eiroyrgeiwn-ndash-anabolh-apogeymatinwn-iatreiwn.

3. Apostolou K, Vogli S, Frountzas M, et al. Upper gastrointestinal cancer management in the COVID-19 era: risk of infection, adapted role of endoscopy, and potential treatment algorithm alterations. $J$ Gastrointest Cancer. 2021;(52):407-413. [CrossRef]

4. McLean RC, Young J, Musbahi A, et al. A single-centre observational cohort study to evaluate volume and severity of emergency general surgery admissions during the COVID-19 pandemic: is there a "lockdown" effect? Int J Surg. 2020;83:259-266. [CrossRef]

5. D’Urbano F, Fabbri N, Koleva Radica M, Rossin E, Carcoforo P. Emergency surgery in COVID-19 outbreak: has anything changed? Single center experience. World $J$ Clin Cases. 2020;8(17):3691-3696. [CrossRef]

6. Moustakis J, Piperidis AA, Ogunrombi AB. The effect of COVID-19 on essential surgical admissions in South Africa: a retrospective observational analysis of admissions before and during lockdown at a tertiary healthcare complex. S Afr Med J. 2020;110(9):910-915. [CrossRef]

7. Nasta AM, Goel R, Kanagavel M, Easwaramoorthy S. Impact of COVID-19 on general surgical practice in India. Indian J Surg. 2020;82(3):259-263. [CrossRef]

8. Gountas I, Hillas G, Souliotis K. Act early, save lives: managing COVID-19 in Greece. Public Health. 2020;187:136-139. [CrossRef]

9. Santoro GA, Grossi U, Murad-Regadas S, et al. DElayed COloRectal cancer care during COVID-19 Pandemic (DECOR-19): global perspective from an International Survey. Surgery. 2021;169(4):796-807. [CrossRef] 